Marquette University

e-Publications@Marquette

Exercise Science Faculty Research and

Publications

Exercise Science, Department of

$10-2020$

\title{
Time-Frequency Analysis of Muscle Activation Patterns in People with Chronic Ankle Instability During Landing and Cutting Tasks
}

Hoon Kim

Marquette University

Rianna M. Palmieri-Smith

University of Michigan - Ann Arbor

Kristof Kipp

Marquette University, kristof.kipp@marquette.edu

Follow this and additional works at: https://epublications.marquette.edu/exsci_fac

\section{Recommended Citation}

Kim, Hoon; Palmieri-Smith, Rianna M.; and Kipp, Kristof, "Time-Frequency Analysis of Muscle Activation Patterns in People with Chronic Ankle Instability During Landing and Cutting Tasks" (2020). Exercise Science Faculty Research and Publications. 187.

https://epublications.marquette.edu/exsci_fac/187 
Marquette University

e-Publications@Marquette

\section{Exercise Sciences Faculty Research and Publications/College of Health} Sciences

This paper is NOT THE PUBLISHED VERSION.

Access the published version via the link in the citation below.

Gait \& Posture, Vol. 82 (October 2020): 203-208. DOI. This article is (C Elsevier and permission has been granted for this version to appear in e-Publications@Marquette. Elsevier does not grant permission for this article to be further copied/distributed or hosted elsewhere without the express permission from Elsevier.

\section{Time-Frequency Analysis of Muscle Activation Patterns in People with Chronic Ankle Instability During Landing and Cutting Tasks}

Hoon Kim

Department of Physical Therapy - Program in Exercise \& Rehabilitation Science, Marquette University, Milwaukee, WI

Riann Palmieri-Smith

School of Kinesiology, University of Michigan, Ann Arbor, MI

Orthopaedic and Rehabilitation Biomechanics Laboratory, University of Michigan, Ann Arbor, MI

Kristof Kipp

Department of Physical Therapy - Program in Exercise \& Rehabilitation Science, Marquette University, Milwaukee, WI 


\section{Abstract}

\section{Background}

People with chronic ankle instability (CAI) exhibit neuromuscular deficits. Previous studies, however, only investigated magnitudes of muscle activation and not the time-frequency domain.

\section{Research question}

Do people with CAI exhibit differences in muscle activation patterns in the time-frequency domain during landing, anticipated cutting, and unanticipated cutting compared to matched controls?

\section{Methods}

Eleven people with CAI and eleven healthy matched controls (CON) performed landing, anticipated cutting, and unanticipated cutting as surface EMG of the lateral gastrocnemius, medial gastrocnemius, fibularis longus, soleus, and tibialis anterior were recorded. The time-frequency domain of surface EMG data was analyzed with wavelet transformations and principal component analysis (PCA), PC scores were compared across group, task, and muscle with three-way ANOVAs.

\section{Results}

The PCA extracted two PCs that captured the overall magnitude (PC1) of wavelet intensities across the timefrequency domain and a shift among the range of frequencies (PC2) where wavelet intensities were most prominent. A main effect for group indicated that people with CAI demonstrated smaller $(p=0.009)$ PC1 scores than people in the CON group across all muscles and tasks. An interaction between group and task indicated that people in the CAI group exhibited smaller $(p=0.041)$ PC2 scores than people in the CON group during only anticipated cutting.

\section{Significance}

People with CAl exhibited neuromuscular deficits in the time-frequency domain of EMG during dynamic tasks. These deficits appear to reflect a neuromuscular strategy characterized by the recruitment of fewer motor units in ankle muscles regardless of task, and an inability to scale the recruitment of motor units in the frequency domain in response to different task demands. Rehabilitation for people with CAl should consider that this population exhibits differences in neuromuscular control that exist not only in the overall magnitudes, but also in the time-frequency domain, of muscle activation patterns.

\section{Keywords}

Emg, Wavelet, Injury, Sprain

\section{Introduction}

Ankle sprains are among the most common injuries in athletes and physically active people [1]. Up to $70 \%$ of the general population has experienced at least one ankle sprain [2]. A previous study also reported that up to $74 \%$ of people with a history of ankle sprains develop chronic ankle instability (CAI), which is a condition characterized by recurring or repeated giving away of the ankle during dynamic activities $[1,3]$. Recurrent ankle sprains are associated with mechanical and functional deficits and become a critical issue in people with CAI because it limits their physical activities [4], and leads to residual symptoms such as pain, swelling, or feeling of giving away [5]. In addition, CAl is associated with the development of ankle osteoarthritis due to damage of the talocrural joint surface $[6,7]$. Furthermore, people with CAI exhibit different neuromuscular strategies, such as altered muscle activations, as they walk or perform sport-related landing or cutting motions $[8,9]$. 
Electromyography (EMG) is an important research tool to investigate the neuromuscular strategies during sportrelated motions, and several studies have used EMG to identify altered neuromuscular strategies of people with CAI compared to people without CAI. [8,[10], [11], [12], [13], [14]]. Previous studies revealed different muscle activation strategies during dynamic tasks, such as landing or cutting, in people with CAI compared to healthy people. For example, in some studies people with CAI exhibit less activation of the fibularis longus, tibialis anterior, medial gastrocnemius, and gluteus medius during a jump land and cut task [11] and diagonal single-leg rebound jumping [14] compared to healthy controls in previous studies. In contrast, a different study showed that people with CAI muscles exhibit greater activation of the medial gastrocnemius, fibularis longus, adductor longus, vastus lateralis, gluteus medius, and gluteus maximus compared to healthy controls, but only during specific time periods of a jump land and cut task [8]. Furthermore, fibularis longus, rectus femoris, tibialis anterior, and soleus muscle activations after initial contact did not differ between people with and without CAI during single leg drop jumps [15]. Since results from previous studies show inconsistent findings in the amplitude of muscle activation during dynamic movements, it is possible that investigating and comparing only the amplitudes of muscle activation of people with and without CAI may not provide adequate insight into neuromuscular deficits.

EMG data in studies that investigate neuromuscular control in people with CAI are frequently filtered and smoothed (e.g., band-pass filtered with cutoff frequencies of 20 and $450 \mathrm{~Hz}$ and smoothed with root mean square algorithms), which removes, and essentially ignores, information about the frequency domain of EMG data and the neuromuscular recruitment strategies of different muscles [16]. Some studies have investigated the time-frequency domain of EMG data in order to differentiate between people with different impairments or knee injury history [[17], [18], [19], [20], [21]]. For example, von Tscharner and Valderrabano (2010) succesfully used time-frequency features of muscle activations to accurately classify people with ankle osteoarthritis and healthy people [19]. However, no study to date has investigated muscle activation characteristics in the timefrequency domain of people with CAI. Given that investigating muscle activation in the time-frequency domain may reveal information about motor unit and muscle recruitment strategies, which are relevant to designing targeted clinical interventions, studying the characteristics of clinically important muscles (e.g., fibularis longus) in this domain in people with CAI seems warranted.

To better understand the neuromuscular strategies used by people with CAl it would be important to investigate the muscle activation patterns in the time-frequency domain. Therefore, the purpose of the current study was to identify differences the time-frequency domain of muscle activation patterns between people with and without CAl during athletic tasks (e.g., landing, anticipated cutting, and unanticipated cutting). We hypothesized that 1) there would be significant differences in the frequencies of muscle activation patterns between people with and without CAI and 2) that these differences would be task-dependent.

\section{Methods}

\subsection{Participants}

Eleven people with CAI $(22.4 \pm 3.2$ years, $1.68 \pm 0.11 \mathrm{~m}, 69.0 \pm 19.1 \mathrm{~kg})$ and 11 healthy people $(22.6 \pm 4.2$ years, $1.74 \pm 0.11 \mathrm{~m}, 66.8 \pm 15.5 \mathrm{~kg}$ ) were recruited for this study. All participants signed an informed consent form that was approved by an Institutional Review Board, which ensured that the research complied with the ethical principles of the Declaration of Helsinki. Inclusion criteria for the CAl group were based on a modified version of the Ankle Instability Instrument, which used nine questions to assess various aspects of a person's history of ankle sprains and associates symptoms [22,23]. The Foot \& Ankle Disability Index (FADI) and FADI-Sport (FADI-S) questionnaires were additionally used to quantify the function and disability in the ankle joint (FADI, CAI group: $90.3 \pm 9.4 \%$, CON: $100 \pm 0 \%$; FADI-S, CAI: $88.6 \pm 9.1 \%$, CON: $100 \pm 0 \%)[24,25]$. Participants from the control 
group were matched to the CAI group based on sex, age, height, weight, and physical activity level, which was assessed via Tegner scores [24].

\subsection{Data collection}

Participants were instrumented with five electromyographical (EMG) sensors (Bagnoli 8-Channel Desktop System, Delsys, Boston, MA, USA). These EMG sensors were attached over the muscle bellies of the lateral gastrocnemius (LG), medial gastrocnemius (MG), fibularis longus ( $F L)$, soleus $(S L)$, and tibialis anterior (TA) after cleaning of skin with an alcohol swab on the EMG attached area. Each participant performed three tasks: 1) double-leg forward jump with single-leg landing, 2) double-leg forward jump with single-leg landing and anticipated cutting, and 3) double-leg forward jumping with single-leg landing and unanticipated cutting. The order of the three tasks remained the same for each participant so that the difficulty increased progressively (e.g., landing, anticipated cutting, unanticipated cutting). For each task, participants were asked to perform the double-leg forward-jump over a $15 \mathrm{~cm}$ box and to land on a force plate (AMTI OR6, Advanced Medical Technology Inc., Watertown, MA, USA). The distance between initial position and the force plate was each participant's leg length which was measured from anterior superior iliac spine and medial malleolus. For the single-leg landing task, participants were asked to land and stabilize their body for 5 seconds. For the single-leg landing and anticipated cutting task, participants were asked to perform a $90^{\circ}$ cut away from their landing leg immediately after landing on the force plate. The single-leg landing and unanticipated cutting task were similar to the anticipated cutting task, but the landing leg and the cutting direction were presented to participants by a visual stimulus that was displayed on a laptop monitor, which was positioned at waist height just behind the force plate, and came on as participants broke a light beam set halfway between the take-off and landing area. Participants were asked to perform between three to five successful trials of each task. Trials were considered successful if participants performed the task according to instructions and landed with their foot entirely on the force plate.

\subsection{Data recording and processing}

Force plate and EMG data were recorded during the stance phase of each task at $1200 \mathrm{~Hz}$. EMG data were amplified by a factor of 1000 . The beginning of stance phase was defined as the point when the vertical ground reaction force (GRF) data exceeded $10 \mathrm{~N}$. The end of the stance phase for the single-leg landing was defined as $200 \mathrm{~ms}$ after the beginning of stance phase, whereas for both cutting tasks it was defined as the point when the GRF fell below $10 \mathrm{~N}$. We excluded one subject in the control group because of technical problems with the GRF. In addition, two participants in the CAI group were not able to perform the unanticipated cutting task.

Therefore, the total number of trials included in the analysis was 183 i.e., 15 less (one CON: 3 tasks $\times 3$ trials; two CAI: 1 task $\times 3$ trials) than if the data set had been complete and included all 198 trials ( 22 subjects $\times 3$ tasks $\times 3$ trials).

Wavelet transformations allow for the simultaneous analysis of EMG signals in the time and frequency domains with various resolutions [20]. The intensity of the EMG signal was calculated with a wavelet intensity analysis in which EMG data was transformed in the time-frequency domain with a set of 11 nonlinearly scaled Cauchy wavelets (w1-w11) [17,20]. The center frequencies of the wavelets were 6.90, 19.29, 37.71, 62.09, 92.36, $128.48,170.39,218.08,271.50,330.63$, and $395.46 \mathrm{~Hz}$ to capture the full range of the EMG signal spectrum [20]. The intensities were time-normalized to make $0-100 \%$ of stance phase (Fig. 1). The intensity from w1 was excluded for further analyses because it was considered to reflect movement artifacts [26]. The total intensities of each wavelet (w2-w11) were compiled into a matrix. The matrix had 915 rows representing (183 trials $\times 5$ muscles) and 10 columns representing total intensity from w2-w11. A principal component analysis was applied to the matrix to find the principal components (PC) that accounted for $90 \%$ of the total variance (VAF). 


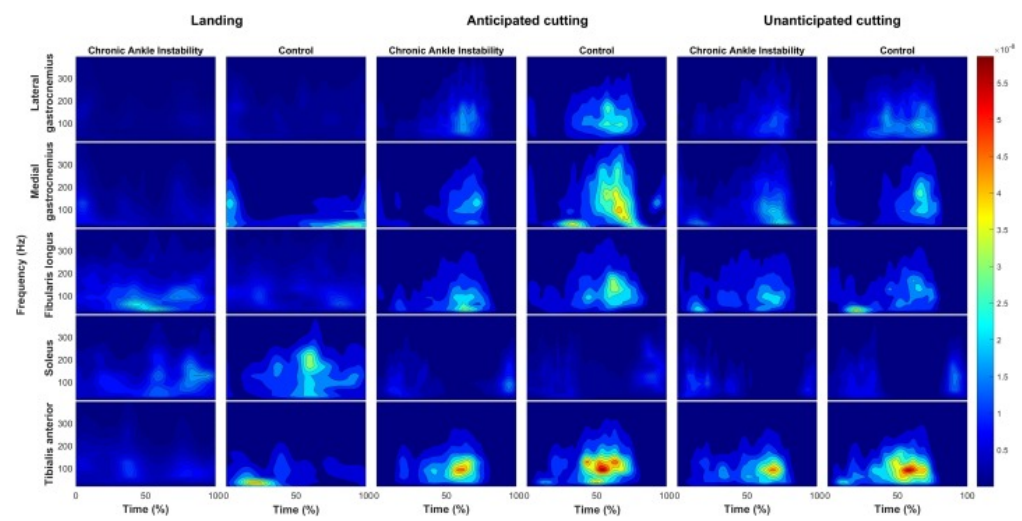

Fig. 1. Time-frequency heatmaps for mean wavelet intensities of the lateral gastrocnemius (first row), medial gastrocnemius (second row), fibularis longus (third row), soleus (fourth row), and tibialis anterior (fifth row) during $200 \mathrm{~ms}$ of the landing task (left two columns) and during the stance phases of the anticipated cutting task (middle two columns) and unanticipated cutting task (right two columns).

For the statistical analysis, the dependent variables were the extracted PC scores. The independent variables were group (CON and CAI), task (landing, anticipated cutting, and unanticipated cutting), and muscles (SL, FL, TA, $M G$, and LG). The Kolmogorov-Smirnov test was used to check the normal distribution of the PC scores. Separate three-way ANOVAs for each PC Score were used to analyze the interactions and main effects of all experimental conditions. Fisher's Least Significant Difference procedure was used during post hoc testing to investigate pairwise differences for any significant interactions or main effects. Two-way interactions not involving group as a factor and main effects other than group were not investigated because they did not directly relate to the purpose of the study (e.g., task $\times$ muscle differences were not of interest). Alpha value was set to 0.05 .

\section{Results}

The PCA extracted two PCs, which accounted for $71 \%$ and $18 \%$ of the total variance in the EMG input data (Fig. 2). The first PC captured variation in the magnitude of the wavelet intensities whereas the second PC captured variation related to a shift in the center frequencies of wavelet intensity.
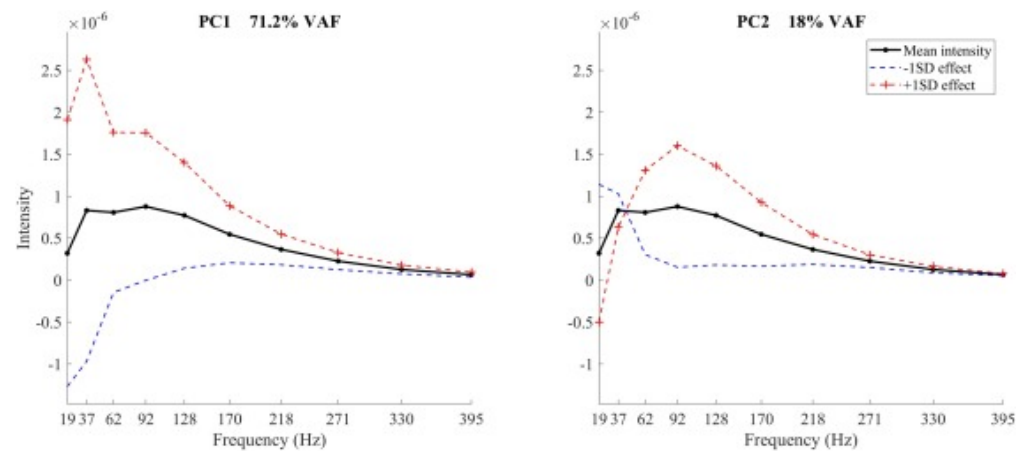

Fig. 2. Variation in wavelet intensity captured by the two principal components (PC). The effects of positive and negative PC scores on wavelet intensities are illustrated by simulating a one standard deviation (1SD) change in the PC on the mean intensity of the EMG data and can be visualized by the dashed lines and the + and symbols, respectively. The effects. VAF - variance accounted for by the given PC.

None of the three-way interactions between group, task, and muscle were significant for either PC (Table 1). In addition, neither of the two-way interactions that included Group as a factor were significant for the first PC. There was, however, a significant $(p=0.041)$ two-way interaction between Group and Task for the second PC (Fig. 3). Post hoc testing revealed that the scores of PC2 were significantly $(p=0.009)$ lower in all muscles of the 
CAI group during the anticipated cutting task only. Lastly, there was also a significant $(p=0.009)$ main effect of group for the scores of the first PC, which showed that the CAI group exhibited lower PC1 scores across all tasks and for all muscles (Fig. 4).

Table 1. Principal component (PC) scores (means and standard deviation) for lateral gastrocnemius (LG), medial gastrocnemius (MG), fibularis longus $(\mathrm{FL})$, soleus $(\mathrm{SOL})$, and tibialis anterior $(\mathrm{TA})$ in people with chronic ankle instability (CAI) and for healthy controls (CON) for the landing (Land), anticipated cutting (Ant), and unanticipated cutting (Unant) tasks.

\begin{tabular}{|l|l|l|l|l|l|l|l|}
\hline & & PC1 score & & & PC2 score & & \\
\hline Muscle & Group & Land & Ant & Unant & Land & Ant & Unant \\
\hline LG & CAI & $-12.60 \pm 1.73$ & $-6.48 \pm 6.84$ & $-7.16 \pm 5.89$ & $-7.72 \pm 1.54$ & $-2.11 \pm 6.31$ & $-3.08 \pm 4.58$ \\
\hline & CON & $-10.87 \pm 2.89$ & $-0.89 \pm 12.95$ & $-0.44 \pm 11.17$ & $-6.88 \pm 2.77$ & $5.14 \pm 13.85$ & $1.08 \pm 7.85$ \\
\hline MG & CAI & $-6.65 \pm 7.21$ & $-2.34 \pm 7.92$ & $0.48 \pm 7.90$ & $-5.92 \pm 3.82$ & $-1.61 \pm 4.12$ & $-1.61 \pm 3.19$ \\
\hline & CON & $10.13 \pm 87.45$ & $16.27 \pm 77.59$ & $-1.30 \pm 7.39$ & $-10.06 \pm 38.11$ & $2.83 \pm 18.50$ & $0.17 \pm 6.01$ \\
\hline FL & CAI & $5.49 \pm 34.98$ & $2.62 \pm 22.47$ & $2.54 \pm 13.36$ & $3.26 \pm 14.91$ & $1.30 \pm 7.81$ & $3.04 \pm 9.33$ \\
\hline & CON & $-4.68 \pm 16.25$ & $1.63 \pm 24.68$ & $3.95 \pm 22.18$ & $-0.84 \pm 13.54$ & $6.67 \pm 24.11$ & $0.01 \pm 15.66$ \\
\hline SOL & CAI & $0.69 \pm 17.88$ & $-8.18 \pm 5.90$ & $-7.97 \pm 5.78$ & $2.53 \pm 14.53$ & $-4.24 \pm 4.68$ & $-3.95 \pm 5.35$ \\
\hline & CON & $7.27 \pm 21.97$ & $-9.08 \pm 4.86$ & $-7.89 \pm 7.54$ & $7.41 \pm 17.23$ & $-4.70 \pm 5.69$ & $-3.99 \pm 6.12$ \\
\hline TA & CAI & $-5.72 \pm 4.29$ & $4.60 \pm 8.78$ & $5.74 \pm 11.10$ & $-2.27 \pm 4.24$ & $7.36 \pm 8.33$ & $5.82 \pm 8.69$ \\
\hline & CON & $8.00 \pm 48.28$ & $11.31 \pm 30.70$ & $13.75 \pm 35.19$ & $-7.06 \pm 11.43$ & $10.58 \pm 19.51$ & $9.82 \pm 23.65$ \\
\hline
\end{tabular}

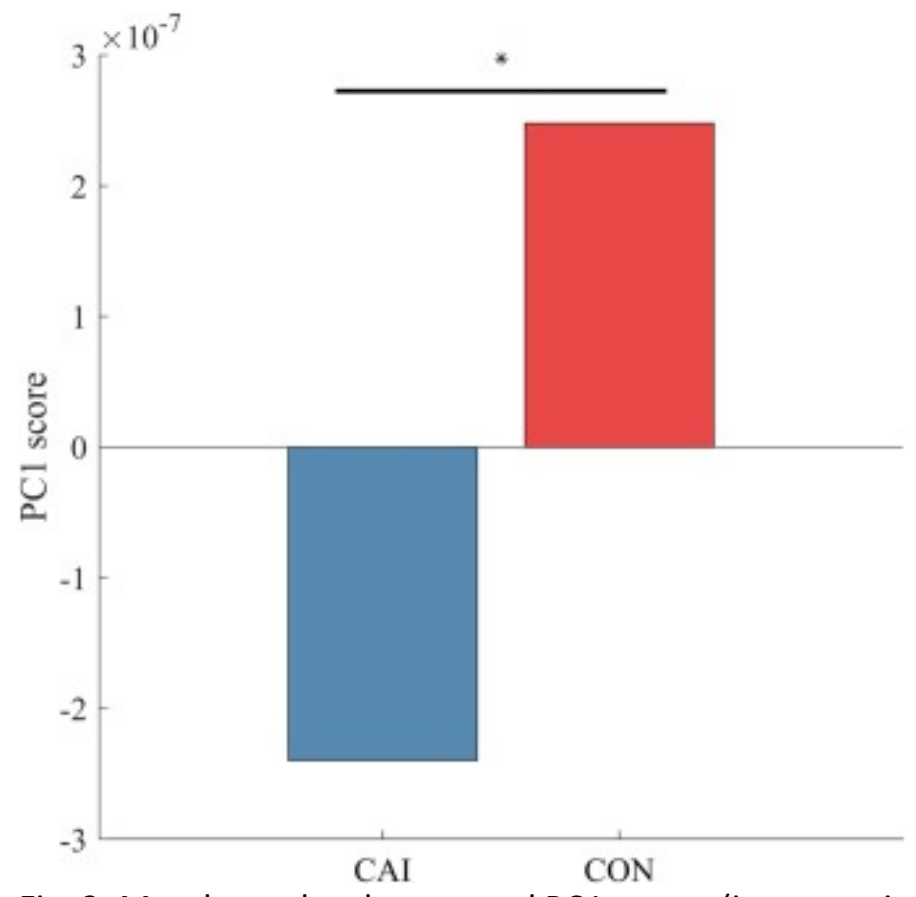

Fig. 3. Muscle- and task-averaged PC1 scores (i.e., magnitude of the wavelet intensities) for people with chronic ankle instability (CAI) and for people in the control group (CON). 




Fig. 4. Muscle-averaged PC2 scores (i.e., shift in center frequencies of wavelet intensities) for people with chronic ankle instability (CAI) and for people in the control group (CON) during the landing, anticipated cutting, and unanticipated cutting task.

\section{Discussions}

The purpose of the current study was to investigate differences in the time-frequency domain of muscle activation patterns between people with and without CAI during athletic tasks (e.g., landing, anticipated cutting, and unanticipated cutting). We hypothesized that people with CAI would exhibit different activation patterns and that these differences would depend on the respective task. The results generally supported our hypotheses in that people with CAl exhibited lower wavelet intensities across all muscles and tasks, and were not able to increase wavelet intensities at higher frequency ranges during the anticipated cutting task.

The main effect for PC1 indicated that compared to the CON group, people with CAI exhibited lower PC1 scores in all muscles and across all tasks. Since PC1 captured the general magnitude of wavelet intensities across all frequencies, people with CAI therefore exhibited lower wavelet intensities across all frequency ranges in all muscles and tasks. People with CAI thus appear to use a neuromuscular strategy characterized by activating ankle muscles at lower wavelet intensities during landing and cutting tasks. Previous studies that investigated neuromuscular function in people with CAI during dynamic movements presented inconsistent findings with respect to the peak amplitude of the smoothed EMG signal in that they show that people with CAl exhibit either less activation of the FL, TA, and MG muscles [11] or more activation of the FL and MG muscles [8] than healthy controls. The intensity of a wavelet provides a good approximation of power of the EMG signal at that respective frequency, is related to changes in the Root Mean Square of EMG, and provides insight into the number of active motor units $[17,19,20]$. The current finding therefore suggests that people with CAI recruit fewer motor units in ankle muscles regardless of task, and is in agreement with research that showed less activation ankle muscles in people with CAI [11]. The current study thus expands on previous results since the wavelet analysis accounts for the intensities across a wide range of activation frequencies, and presumably motor units [20]. Collectively, these findings suggest CAI rehabilitation may need to include resistance training exercises that aim to increase muscle force production across a large range of activation frequencies so that all motor units are adequately trained. 
The group by task interaction effect for PC2 indicated that people with CAl exhibited similar PC2 scores across all tasks, whereas people in the CON group did not. Specifically, post hoc testing revealed that people in the CON group exhibited greater PC2 scores than the CAI group during the anticipated cutting task. Since PC2 captured a shift among the range of frequencies where the wavelet intensities were most prominent, the increase in PC2 scores in the CON group suggests that this group exhibited an increase in wavelet intensities at higher frequency ranges during the anticipated cutting task. In other words, people with CAI seemed unable to increase wavelet intensities in higher frequency ranges during anticipated cutting. The frequency domain of a muscle's activation profile is influenced by the recruitment of different motor unit types because the conduction velocity of motor units differs based on the electric properties of the motor units i.e., faster motor units exhibit higher conduction velocities and greater wavelet frequencies during muscle activation than slower motor units $[27,28]$. Since cutting motions require rapid muscle activation, these movements also likely require that motor units are recruited at higher frequencies [29]. Based on the analysis of PC2 scores, it thus appears that people with CAI are not able to recruit motor units during the anticipated cutting task in the same manner as people in the CON group. This finding may indicate that people with CAI are not able to adequately scale the recruitment of motor units in the frequency domain in response to different task demands. Given that movements are controlled with not only the intensity of muscle activations but also speed of motor unit recruitment, which suggests that people with CAI may stem from incapacity to scale frequency of muscle activations during the dynamic tasks. Perhaps, performing resistance training exercises during CAI rehabilitation at fast velocities may facilitate the recruitment and increase the firing rate of faster motor units and thus improve the capacity to shift wavelet intensity towards higher frequency during muscle activation in people with CAI.

There are several limitations with the current study. First, the FADI and FADI-Sport questionnaires were used to assess self-reported ankle function in the current study. Other studies, however, have used questionnaires such as the Cumberland Ankle Instability Tool [3]. Although the Cumberland Ankle Instability Tool is commonly used, FADI and FADI-Sport are considered to adequately describe functional deficits in people with CAI [25]. In addition to the FADI and FADI-Sport, we also used another questionnaire to evaluate the history and symptoms of ankle sprain [22], which enhanced the CAI inclusion criteria. Second, the current study did not include a group of "copers" (i.e., people who have a history of lateral ankle sprain but have no recurrent ankle sprains or functional deficits). A previous study revealed that copers exhibit different sensorimotor function than people with CAI [30]. For this reason, recruiting a copers group in addition to people with CAI and healthy controls may provide better clinical insights and more detailed information about the spectrum of functional deficits in people with CAI. Third, only the landing and stance phases during three tasks were analyzed in this study. However, the phase immediately before foot contact can provide information about preparatory strategies during the dynamic tasks and has been widely analyzed in previous CAI studies [31,32]. Therefore, analyzing muscle activation in the frequency domain during the preparatory phase of dynamic tasks in people with CAl in future studies may also provide additional information. Fourth, we only collected and analyzed EMG of distal muscles (i.e., at the ankle joint). Including proximal muscles for time-frequency analysis in future studies may also be useful because previous research suggests that people with CAI also exhibit different activation patterns of proximal muscles, such as the vastus lateralis and gluteus maximus [8]. Lastly, the sample size of people with CAI in this study is relatively small, which may suggest that the results and interpretations should be considered as preliminary and should be replicated in a larger sample.

To our knowledge, this study is the first study to investigate the time-frequency domain of muscle activation patterns in people with CAI. There are several clinical implications of the results from the current study. We found that people with CAI exhibited lower wavelet intensities across all tasks and all muscles and did not change the muscle activation in the time-frequency domain in response to different tasks. Collectively, people with CAl appear to activate fewer motor units in all ankle muscles we analyzed during all studied dynamic tasks, and recruit slower motor units within all analyzed ankle muscles during anticipated cutting. Given that people 
with CAI exhibited neuromuscular deficits in both wavelet intensity and frequency of ankle muscle activations in the current study, rehabilitation to improve neuromuscular control and decrease risk of recurrent injuries in people with CAI will likely need to include exercises that focus on the velocity component of contraction (e.g., anticipated and unanticipated tasks with various moving directions) in addition to the intensity component (e.g., loads) of contraction.

\section{Conclusion}

People with CAI exhibited muscle activation patterns characterized by differences in the time-frequency domain compared to healthy people. Specifically, people with CAI activated all ankle muscles with lower wavelet intensities across the entire frequency spectrum, regardless of task. In addition, people with CAI did not exhibit an increase in wavelet intensity in higher ranges of the frequency spectrum during the anticipated cutting task. These findings suggest that rehabilitation efforts for people with CAI should consider that this population exhibits differences in neuromuscular control that exist not only in the overall magnitudes, but also in the timefrequency domain, of muscle activation patterns.

\section{Declaration of Competing Interest}

The authors report no declarations of interest.

\section{Acknowledgments}

The authors would like to acknowledge assistance from the National Institutes of Health (T32 HD007422-20) and a Research Stipend from the NIDRR.

\section{References}

[1] M.M. Herzog, Z.Y. Kerr, S.W. Marshall, E.A. Wikstrom. Epidemiology of ankle sprains and chronic ankle instability. J. Athl. Train., 54 (6) (2019), pp. 603-610. https://www.ncbi.nlm.nih.gov/pubmed/31135209

[2] G.D. McKay, P.A. Goldie, W.R. Payne, B.W. Oakes. Ankle injuries in basketball: injury rate and risk factors. Br. J. Sports Med., 35 (2) (2001), pp. 103-108. https://www.ncbi.nlm.nih.gov/pubmed/11273971

[3] P.A. Gribble, E. Delahunt, C.M. Bleakley, B. Caulfield, C.L. Docherty, D.T. Fong, et al. Selection criteria for patients with chronic ankle instability in controlled research: a position statement of the International Ankle Consortium. J. Athl. Train., 49 (1) (2014), pp. 121-127. https://www.ncbi.nlm.nih.gov/pubmed/24377963

[4] T.J. Hubbard, E.A. Wikstrom. Ankle sprain: pathophysiology, predisposing factors, and management strategies. Open Access J. Sports Med., 1 (2010), pp. 115-122. https://www.ncbi.nlm.nih.gov/pubmed/24198549

[5] J. Hertel. Functional anatomy, Pathomechanics, and pathophysiology of lateral ankle instability. J. Athl. Train., 37 (4) (2002), pp. 364-375. https://www.ncbi.nlm.nih.gov/pubmed/12937557

[6] B. Hintermann, A. Boss, D. Schafer. Arthroscopic findings in patients with chronic ankle instability. Am. J. Sports Med., 30 (3) (2002), pp. 402-409. https://www.ncbi.nlm.nih.gov/pubmed/12016082

[7] E.A. Wikstrom, T. Hubbard-Turner, P.O. McKeon. Understanding and treating lateral ankle sprains and their consequences: a constraints-based approach. Sports Med., 43 (6) (2013), pp. 385-393. https://www.ncbi.nlm.nih.gov/pubmed/23580392

[8] H. Kim, S.J. Son, M.K. Seeley, J.T. Hopkins. Altered movement strategies during jump landing/cutting in patients with chronic ankle instability. Scand. J. Med. Sci. Sports, 29 (8) (2019), pp. 1130-1140. https://www.ncbi.nlm.nih.gov/pubmed/31050053

[9] S.J. Son, H. Kim, M.K. Seeley, J.T. Hopkins. Altered walking neuromechanics in patients with chronic ankle instability. J. Athl. Train., 54 (6) (2019), pp. 684-697. https://www.ncbi.nlm.nih.gov/pubmed/31162941 
[10] J. Hertel, R.O. Corbett. An updated model of chronic ankle instability. J. Athl. Train., 54 (6) (2019), pp. $572-$ 588. https://www.ncbi.nlm.nih.gov/pubmed/31162943

[11] S.J. Son, H. Kim, M.K. Seeley, J.T. Hopkins. Movement strategies among groups of chronic ankle instability, Coper, and control. Med. Sci. Sports Exerc., 49 (8) (2017), pp. 1649-1661. https://www.ncbi.nlm.nih.gov/pubmed/28350716

[12] C.C. Herb, K. Grossman, M.A. Feger, L. Donovan, J. Hertel. Lower extremity biomechanics during a dropvertical jump in participants with or without chronic ankle instability. J. Athl. Train., 53 (4) (2018), pp. 364-371. https://www.ncbi.nlm.nih.gov/pubmed/29667844

[13] M.A. Feger, L. Donovan, J.M. Hart, J. Hertel. Lower extremity muscle activation in patients with or without chronic ankle instability during walking. J. Athl. Train., 50 (4) (2015), pp. 350-357. https://www.ncbi.nlm.nih.gov/pubmed/25562453

[14] S. Kunugi, A. Masunari, T. Koumura, A. Fujimoto, N. Yoshida, S. Miyakawa. Altered lower limb kinematics and muscle activities in soccer players with chronic ankle instability. Phys. Ther. Sport, 34 (2018), pp. 28-35. https://www.ncbi.nlm.nih.gov/pubmed/30144785

[15] E. Delahunt, K. Monaghan, B. Caulfield. Changes in lower limb kinematics, kinetics, and muscle activity in subjects with functional instability of the ankle joint during a single leg drop jump. J. Orthop. Res., 24 (10) (2006), pp. 1991-2000. https://www.ncbi.nlm.nih.gov/pubmed/16894592

[16] J.M. Wakeling, K. Uehli, A.I. Rozitis. Muscle fibre recruitment can respond to the mechanics of the muscle contraction. J. R. Soc. Interface, 3 (9) (2006), pp. 533-544. https://www.ncbi.nlm.nih.gov/pubmed/16849250

[17] C. Jewell, J. Hamill, V. von Tscharner, K.A. Boyer. Altered multi-muscle coordination patterns in habitual forefoot runners during a prolonged, exhaustive run. Eur. J. Sport Sci., 19 (8) (2019), pp. 1062-1071. https://www.ncbi.nlm.nih.gov/pubmed/30732537

[18] M. Mohr, V. von Tscharner, C.A. Emery, B.M. Nigg. Classification of gait muscle activation patterns according to knee injury history using a support vector machine approach. Hum. Mov. Sci., 66 (2019), pp. 335-346. https://www.ncbi.nlm.nih.gov/pubmed/31146192

[19] V. von Tscharner, V. Valderrabano. Classification of multi muscle activation patterns of osteoarthritis patients during level walking. J. Electromyogr. Kinesiol., 20 (4) (2010), pp. 676-683. https://www.ncbi.nlm.nih.gov/pubmed/20005128

[20] V. von Tscharner. Intensity analysis in time-frequency space of surface myoelectric signals by wavelets of specified resolution. J. Electromyogr. Kinesiol., 10 (6) (2000), pp. 433-445. https://www.ncbi.nlm.nih.gov/pubmed/11102846

[21] G. Kuntze, V. von Tscharner, C. Hutchison, J.L. Ronsky. Multi-muscle activation strategies during walking in female post-operative total joint replacement patients. J. Electromyogr. Kinesiol., 25 (4) (2015), pp. 715-721. https://www.ncbi.nlm.nih.gov/pubmed/25908584

[22] E.D. McVey, R.M. Palmieri, C.L. Docherty, S.M. Zinder, C.D. Ingersoll. Arthrogenic muscle inhibition in the leg muscles of subjects exhibiting functional ankle instability. Foot Ankle Int., 26 (12) (2005), pp. 10551061. https://www.ncbi.nlm.nih.gov/pubmed/16390639

[23] C.L. Docherty, B.M. Gansneder, B.L. Arnold, S.R. Hurwitz. Development and reliability of the ankle instability instrument. J. Athl. Train., 41 (2) (2006), pp. 154-158. https://www.ncbi.nlm.nih.gov/pubmed/16791299

[24] K. Kipp, R.M. Palmieri-Smith. Differences in kinematic control of ankle joint motions in people with chronic ankle instability. Clin. Biomech. Bristol Avon (Bristol, Avon), 28 (5) (2013), pp. 562-567. https://www.ncbi.nlm.nih.gov/pubmed/23601918

[25] M.N. Houston, J.M. Hoch, M.C. Hoch. Patient-reported outcome measures in individuals with chronic ankle instability: a systematic review. J. Athl. Train., 50 (10) (2015), pp. 1019-1033. https://www.ncbi.nlm.nih.gov/pubmed/26332028

[26] S. Conforto, T. D'Alessio, S. Pignatelli. Optimal rejection of movement artefacts from myoelectric signals by means of a wavelet filtering procedure. J. Electromyogr. Kinesiol., 9 (1) (1999), pp. 47-57. https://www.ncbi.nlm.nih.gov/pubmed/10022561 
[27] J.M. Wakeling, D.A. Syme. Wave properties of action potentials from fast and slow motor units of rats. Muscle Nerve, 26 (5) (2002), pp. 659-668. https://www.ncbi.nlm.nih.gov/pubmed/12402288

[28] F. Buchthal, C. Guld, P. Rosenfalck. Innervation zone and propagation velocity in human muscle. Acta Physiol. Scand., 35 (2) (1955), pp. 174-190. https://www.ncbi.nlm.nih.gov/pubmed/13301862

[29] S.S. Lee, M. de Boef Miara, A.S. Arnold, A.A. Biewener, J.M. Wakeling. Recruitment of faster motor units is associated with greater rates of fascicle strain and rapid changes in muscle force during locomotion. J. Exp. Biol., 216 (Pt 2) (2013), pp. 198-207. https://www.ncbi.nlm.nih.gov/pubmed/22972893

[30] E.A. Wikstrom, M.D. Tillman, T.L. Chmielewski, J.H. Cauraugh, K.E. Naugle, P.A. Borsa. Discriminating between copers and people with chronic ankle instability. J. Athl. Train., 47 (2) (2012), pp. 136-142. https://www.ncbi.nlm.nih.gov/pubmed/22488278

[31] H. Minoonejad, M. Karimizadeh Ardakani, R. Rajabi, E.A. Wikstrom, A. Sharifnezhad. Hop stabilization training improves neuromuscular control in college basketball players with chronic ankle instability: a randomized controlled trial. J. Sport Rehabil., 28 (6) (2019), pp. 576-583. https://www.ncbi.nlm.nih.gov/pubmed/29952698

[32] A. Rosen, C. Swanik, S. Thomas, J. Glutting, C. Knight, T.W. Kaminski. Differences in lateral drop jumps from an unknown height among individuals with functional ankle instability. J. Athl. Train., 48 (6) (2013), pp. 773-781. https://www.ncbi.nlm.nih.gov/pubmed/23952040 